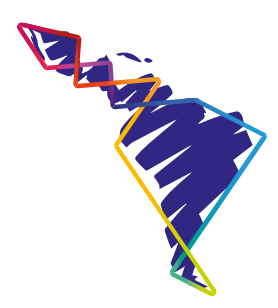

\title{
Inclusión digital de la persona adulta mayor: Una revisión documental
}

\author{
Digital inclusion of the elderly: a \\ literary review
}

\section{Inclusão digital dos idosos: uma revisão documental}

\author{
Jafet Darío M ora-Chavarría ${ }^{1}$ \\ Rodolfo Antonio González-M atamoros²
}

\begin{abstract}
Resumen:
El presente artículo brinda un análisis del estado actual de la inclusión digital, la accesibilidad, y el uso de las herramientas tecnológicas por parte de las personas adultas mayores. Mediante una investigación documental, y un método de investigación de carácter cualitativo con diseño descriptivo donde se seleccionan diferentes estudios científicos para un conocimiento sobre el objeto de estudio. Dentro de los principales resultados, es posible rescatar que los recursos tecnológicos forman parte en el diario vivir del ser humano. Por ello se vuelve indispensable tomar en cuenta a las personas adultas tardías para una capacitación en el uso de dichos recursos, para que, de esta manera, puedan solventar y gestionar actividades necesarias en su vida cotidiana, es decir, que consigan utilizar aplicaciones en teléfonos inteligentes para lograr obtener diferentes oportunidades que se relacionan con servicios de distintas instituciones, acceso a empleo y educación. Además, se consigue identificar que la brecha digital en este conglomerado mantiene sus bases en el factor edad, lo que genera una división significativa entre personas nativas e inmigrantes digitales. Se concluye que el Estado y las instituciones públicas deben atender las necesidades y demandas de la población adulta mayor en materia de educación, así como en tecnologías de la información y la comunicación.
\end{abstract}

Recibido: 6-8-2021 - Aceptado: 5-10-2021

1 Estudiante de Bibliotecología/Orientación (UNA). Asistente del Instituto Regional de Estudios en Sustancias Tóxicas, Universidad Nacional de Costa Rica. Correo electrónico jafet.mora.chavarria@est.una.ac.cr (D) https://orcid.org/0000-0002-7281-0489

2 Estudiante de Bibliotecología (UNA)/Administración Pública (UCR). Asistente de la Biblioteca Especializada en Ciencias Sociales, Universidad Nacional de Costa Rica. Correo electrónico: rodolfo.gonzalez.matamoros@ est.una.ac.cr (D) https://orcid.org/0000-0002-0025-8998 
Palabras claves: Persona adulta mayor, inclusión digital, herramientas tecnológicas, accesibilidad.

\begin{abstract}
This article provides an analysis of the current state of digital inclusion, accessibility, and the use of technological tools by older adults. Through literary research, and a research method of qualitative character with descriptive design wherein different scientific studies are selected to gather knowledge about the object of study. Among the main results, it is possible to rescue that technological resources are part of the daily life of human beings. That is why it becomes essential to take into account the elderly for training in the use of these resources, so that in this way they can solve and manage necessary activities in their daily life, that is, so that they can use applications on smartphones to obtain different opportunities that are related to services from different institutions, access to employment and education. In addition, it is possible to identify that the digital divide in this conglomerate maintains its bases in the age factor, which generates a significant division between digital natives and immigrants. It is concluded that the State and public institutions must meet the needs and demands of the elderly population with regard to education, as well as in information and communication technologies.
\end{abstract}

Keywords: Elderly, digital inclusion, technological tools, accessibility

\title{
Resumo
}

Este artigo apresenta uma análise do estado atual da inclusão digital, acessibilidade e uso de ferramentas tecnológicas por idosos. Por meio da pesquisa documental, é um método de pesquisa qualitativa com desenho descritivo onde são selecionados diferentes estudos científicos para o conhecimento sobre o objeto de estudo. Dentre os principais resultados, é possível resgatar que os recursos tecnológicos fazem parte do cotidiano do ser humano. Por isso, torna-se imprescindível levar em consideração os adultos tardios para o treinamento no uso desses recursos, para que desta forma possam resolver e gerenciar as atividades necessárias ao seu dia a dia, ou seja, que possam utilizar aplicativos em smartphones para obter diferentes oportunidades que estão relacionadas a serviços de diferentes instituições, acesso ao emprego e educação. Além disso, é possível identificar que a exclusão digital neste conglomerado mantém suas bases no fator idade, o que gera uma divisão significativa entre nativos e imigrantes digitais. Conclui-se que o Estado e as instituições públicas devem atender às necessidades e demandas da população idosa na educação, bem como nas tecnologias de informação e comunicação.

Palavras-chave: Idoso, inclusão digital, ferramentas tecnológicas, acessibilidade 


\section{Introducción}

A través del tiempo, la población adulta mayor ha sido considerada como un grupo vulnerable, debido a las características propias de la vejez. Por su parte, el envejecimiento poblacional es una realidad que enfrentan actualmente los países de América Latina, para 2050 se proyecta que la cantidad de personas adultas mayores se constituya como la cuarta parte de la población total mundial (Huenchuan, 2019). Para ese entonces, la sociedad envejecida será una problemática con muchas aristas por resolver, tanto para los Estados como para las organizaciones y la sociedad.

Además, el auge de las tecnologías de la información y la comunicación (en adelante TIC) toma mayor fuerza, dando paso a la sociedad digital y, por lo tanto, a la era del conocimiento. Ambas situaciones se acrecientan con el pasar de los años, lo que supone una brecha entre la población que posee conocimientos en TIC y aquella que debe adaptarse a estos cambios abruptos. Con el contexto anterior, este artículo de investigación tiene como objetivo general analizar el uso de las herramientas tecnológicas en la población adulta mayor para el conocimiento en relación con el acceso a la conectividad y la inclusión digital de estas personas en la sociedad.

Como objetivos específicos se pretende: a) Identificar, mediante la documentación científica, el uso de los recursos tecnológicos por parte de las personas adultas mayores para un análisis de la situación actual. b) Describir el acceso a la conectividad tomando en cuenta los factores de la brecha digital y los sujetos nativos e inmigrantes digitales para un contraste entre el uso de las herramientas tecnológicas y su accesibilidad. c) Verificar la inclusión digital de la población adulta mayor, que se encuentra en la etapa de envejecimiento, por medio de la inclusión educativa para la promoción de igualdad social.

\section{Antecedentes}

Los recursos tecnológicos como detonantes de aprendizaje en las personas adultas mayores han sido investigados por diversas autorías que validan y argumentan el tema con sus propias investigaciones, por lo tanto, se hacen diferentes consultas en distintas fuentes, lo cual permite profundizar en los saberes y la realidad en la que se centra este estudio. Corrales y Sánchez (2018), estudiantes de posgrado en Gerontología de la Universidad de Costa Rica, desarrollan una investigación titulada "El aprendizaje cognoemocional en un grupo de personas mayores". El estudio tiene como objetivo ofrecer una mejor atención integral a las personas adultas mayores, así como un fortalecimiento de su vida desde el área cognitiva y por medio de diferentes redes de apoyo. 
Es mediante la orientación, que se atiende a las personas de la tercera edad, con el apoyo de una fundamentación gerontológica. Su proyecto se basó en trabajar con 10 personas que pertenezcan a la comunidad estudiantil del Programa de Atención Integral de la Persona Adulta Mayor (PAIPAM) de la Universidad Nacional de Costa Rica. El área metodológica se basó en la disciplina orientadora. La argumentación teórica sobre las diferentes áreas del círculo de bienestar integral es el eje fundamental para el desarrollo y diseño de un curso que permitiese abordar la atención mediante la logoterapia, ludoterapia, risoterapia, abrazo terapia y la autoestima. Dentro de sus resultados y conclusiones, se brinda un fortalecimiento efectivo entre cada participante, del mismo modo logran obtener habilidades comunicativas y de expresión, por último, se potencia la autoestima y la autorrealización.

En el año 2019, Rivera y Urbina, en su tesis para optar por el grado de Licenciatura en Educación Comercial en la Escuela de Secretariado de la Universidad Nacional de Costa Rica, titulan su investigación como "Proceso de alfabetización tecnológica en los adultos y adultos mayores participantes del proyecto de extensión en manejo básico de oficinas de la carrera Educación Comercial". Su objetivo de estudio se basó en analizar un proceso de alfabetización tecnológica como proyecto de extensión en manejo básico de oficinas, siendo un aporte significativo en las actividades de sus vidas cotidianas.

Su método de investigación fue cualitativo con diseño fenomenológico, el cual busca describir un fenómeno desde las experiencias y percepciones de los sujetos. Por otra parte, dentro de los instrumentos de recolección de datos que se utilizan está la entrevista, es mediante esta que logran obtener resultados para un objetivo de su estudio. La solución que se brinda en el estudio es mantener el proyecto activo para que, de esta manera, se siga alfabetizando y enseñando a más sujetos adultos mayores con competencias digitales, manteniendo el servicio sin fin de lucro para que pueda ser accesible a toda la población que muestre interés.

Posteriormente, en el año 2020, Flores desarrolla un estudio en la Universidad San Martin de Porres en Perú, titulado: "La alfabetización digital en el público adulto mayor: Un acercamiento desde la comunicación de las relaciones públicas en Perú". Su principal objetivo se centra en identificar, conocer, entender y analizar cómo funciona la comunicación digital en los procesos comunicativos que se originan entre los seres humanos. En su marco teórico definen conceptos como la sociedad de la información, alfabetización digital y el público adulto mayor. La metodología del estudio se basó en un análisis documental de las instituciones del sector público del estado peruano. Las variables de análisis definidas son: "inclusión digital", "alfabetización digital", "educación virtual" y "personas adultas mayores". 
Los resultados arrojados en el estudio muestran que existe un incremento de la esperanza de vida a nivel mundial, por razón de los avances tecnológicos y la ciencia médica. Se denota que la ley de la persona adulta mayor no arroja ningún dato relacionado con la importancia que tiene la alfabetización digital en las personas adultas de la tercera edad, siendo el mismo resultado en el Plan Nacional para las personas adultas mayores. Por último, dentro de las conclusiones que se presentan en la investigación, se muestra que no existe una inclusión en la comunicación pública entre las organizaciones centrales y el público adulto mayor de Perú. Así mismo, alude que estas personas deben hacerse visibles, ya que son capaces de construir sus propios significados digitales y fortalecer, así, la inclusión.

Un estudio realizado en el año 2021 por Benavides y Chipana, titulado "Competencias digitales en adultos mayores y acceso a la justicia: Una revisión sistemática" con el objetivo de mostrar una visión del estado con base a las competencias digitales en la persona adulta mayor, así como la importancia de su inclusión digital. Se estudia la producción científica en revistas indexadas. Las bases de datos consultadas para la revisión documental fueron: Scopus, Scielo, Redalyc, Dialnet y Google Scholar.

En el análisis de datos y discusión se menciona que algunas de las competencias básicas que presentan las personas adultas mayores son las competencias digitales, las cuales son necesarias para el desarrollo de actividades en su vida cotidiana. El Estado cumple, vela y promueve políticas públicas y leyes que atiendan la inclusión, la misma se muestra mediante programas de capacitación y estrategias para el desarrollo de competencias digitales en las personas que se ubiquen en la adultez tardía. En el artículo se concluye que el analfabetismo digital se relaciona con la escasez de competencias digitales que dificulta la accesibilidad a los servicios públicos. Sin embargo, el Estado de Perú debe seguir atendiendo el reto de la inclusión de las personas adultas mayores y dotarlas de competencias y conocimientos que favorezcan su participación, al igual que el resto de la sociedad.

\section{Justificación}

Este artículo de investigación es valioso en la medida en que se da a conocer, mediante la investigación de fuentes documentales, la forma en que la población adulta mayor está utilizando los recursos tecnológicos. Por otra parte, también se brinda un panorama general sobre conectividad e inclusión digital de este segmento poblacional en la sociedad. Es importante conocer este tipo de información, puesto que la Política Nacional de Envejecimiento y Vejez, en su cuarta línea estratégica, plantea una acción relacionada con "facilitar el acceso de las personas adultas mayores a aprender y usar las nuevas tecnologías de la información y la 
comunicación" (Consejo Nacional de la Persona Adulta Mayor, 2013, p. 17). Por lo tanto, se daría a conocer si realmente el Estado está garantizando esta facilidad y si existe un aprovechamiento por parte de la población adulta mayor.

Debido a la naturaleza de la investigación, resulta factible y realizable. Se trata de un estudio de interés social, ya que brinda un acercamiento a la situación que viven las personas adultas mayores actualmente. En lo que respecta a su alcance, se espera determinar, de forma clara, la utilización que se está haciendo de los recursos tecnológicos, su conectividad y un breve análisis sobre la inclusión digital de esta población respecto a los demás grupos etarios.

\section{Referente conceptual}

Los recursos tecnológicos forman parte de los grandes avances tecnológicos que ha tenido que enfrentar la humanidad, por lo tanto, es muy importante hacer hincapié en su definición para que, de esta manera, se pueda comprender el contexto que tienen en el diario vivir de los seres humanos que las utilizan. Según Mandl, Schnurer y Winkler (2004, citados por Ruiz, 2015) "son instrumentos o acciones técnicas, mentales u organizacionales, que inician o apoyan un proceso de gestión del conocimiento, es decir, facilitan la fluidez de la información y el conocimiento en las organizaciones".

A su vez, su impacto en la sociedad ha sido muy característico, por su apoyo en las actividades que se llevan a cabo en instituciones públicas y privadas, así como un medio de comunicación entre distintas personas a nivel nacional e internacional. Según Arias, Sandía y Mora (2012), "el empleo de las herramientas tecnológicas interactivas como soporte de la didáctica, ha generado demandas de estrategias que faciliten y orienten su uso en la educación interactiva a distancia" (p. 22). Por consiguiente, con el pasar de los años, la tecnológica va teniendo un fuerte impacto con singulares repercusiones en diferentes áreas, tales como la social, económica, educativa, cultural, entre otras.

Por otra parte, dentro de las herramientas digitales en las que se desarrolla este estudio está la computadora. Con el pasar de los años ha sido una herramienta esencial para labores empresariales y cotidianas de las personas, ya que les facilita el acceso a diferentes plataformas que ayudan a la gestión ordinaria. McLuhan (2013) las define como "una computadora es una máquina digital y sincrónica, con cierta capacidad de cálculo numérico y lógico, controlada por un programa almacenado y con posibilidad de comunicación con el mundo exterior" (p. 2). 
Aunado a lo anterior, es evidente que las computadoras son una herramienta muy útil para los seres humanos, un instrumento que favorece la rapidez por medio de los servicios en línea o digitales. Las nuevas generaciones, en su educación, van teniendo un implemento de conocimientos basados en este aparato tecnológico, lo que les permite poderse desarrollar aún mejor en el mundo tan sublime en actualizaciones científicas y tecnológicas. Sin embargo, las personas adultas mayores también pueden aprender y utilizar estas herramientas, lo cual resultaría un aporte significativo para la comunicación con sus familiares, pares y amistades.

El celular o smartphone son herramientas que se han incursionado mediante las diferentes revoluciones industriales, forman parte de la vida de las personas en la actualidad. Es un instrumento que ha ido reemplazando diferentes objetos antiguos que en su momento fueron de mucha utilidad para el ser humano. Según Rodríguez, Hernández, Torno, García y Rodríguez (2005), "Los sistemas de telefonía celular son sistemas de comunicaciones móviles en los cuales la zona o territorio en que se brinda el servicio (área de cubrimiento) se divide en celdas (células) ...” (p. 4).

Su impacto ha sido fundamental para la comunicación de las personas en distintas áreas geográficas, pues es un aparato que permite realizar llamadas y diferentes funciones, y brinda una mayor eficacia en las gestiones rutinarias. En la antigüedad, las personas solían comunicarse por medio de epístolas que debían ser redactadas y enviadas con un debido formato. No obstante, con la llegada de los celulares, las personas suelen tener una comunicación más personalizada y rápida, esto mediante las llamadas de voz, videollamadas, mensajes de texto y mensajes de voz. Según el Sistema Nacional de Vigilancia Epidemiológica Fitosanitaria de México (2011):

Los Smartphones, o teléfonos inteligentes, son pequeños dispositivos que integran funcionalidades de teléfono móvil con las funcionalidades más comunes de un PDA (asistente digital personal), además permiten a los usuarios almacenar información, enviar y recibir mensajes, E-mail e instalar programas. (p.3)

A pesar de que ser un beneficio para el ser humano, a su vez es desigual, por el motivo de que no todas las personas pueden acceder a este tipo de aparatos, con los avances en la tecnología y los procesos de desarrollo tecnológico y científico, las entidades comerciantes van creando variedades de herramientas con el fin de que puedan ser accesibles para la mayoría de las personas, sin embargo, lamentablemente, debido a las condiciones socioeconómicas de algunas personas, su posibilidad es nula.

La brecha digital es entendida por Ramírez y Sepúlveda (2018) "como la diferencia que existe entre aquellas personas que tienen acceso a las herramientas TIC y 
aquellas que no" (p. 93). Es importante entender la brecha digital como una desigualdad en el acceso a tecnologías de la información y la comunicación que puede deberse a factores económicos, generacionales y geográficos que interfieren entre el individuo y la herramienta. Para el caso de este estudio, es necesario referirse a inmigrantes y personas nativas digitales.

Por su parte, la definición de persona nativa digital es entendida por Saavedra (2014) "un sujeto con capacidades para realizar procesos simultáneos de forma rápida, preferir el lenguaje gráfico al textual y con habilidades para maniobrar de forma ágil un amplio abanico de recursos tecnológicos" (p. 43). El término fue acuñado por Marc Prensky en 2001, lo utilizó para referirse a un individuo que ha experimentado gran acercamiento a las TIC, por el hecho de haber nacido en la era digital. Por otra parte, migrantes digitales son aquellas personas que se vieron en la necesidad de desarrollar competencias digitales para adaptarse al entorno actual.

Esto coloca a la población adulta mayor en la posición de inmigrante digital, es decir, quienes han debido adaptarse a un mundo donde la economía es digital y se encuentra basada en conocimiento, constituyéndose este último elemento como la mayor ventaja competitiva de que disponen las organizaciones. Lo anterior, producto de la transformación digital que invade todos los rincones profesionales, educativos, laborales, económicos y sociales de la actividad humana; direccionando el accionar de las personas y las organizaciones hacia un aumento significativo de su productividad.

Por lo tanto, resulta indispensable comprender el término "conectividad". Plantea la Organización para la Cooperación y el Desarrollo Económicos (2020) que es necesario "Impulsar la conectividad y la adopción de internet es un primer paso para aprovechar las oportunidades de aprendizaje que aportan las nuevas tecnologías en América Latina" (p. 12). Básicamente la conectividad responde a la capacidad de un dispositivo para establecer una conexión con otro, en este caso, es mediante internet que se posibilita conectar los dispositivos tecnológicos con el mundo digital.

La inclusión digital es un concepto amplio y peculiar, algunos Estados lo están visualizando como una política pública dentro del marco jurídico, un derecho humano, por ello es trascendental definirlo para la comprensión de los saberes conceptuales de este estudio. Mori (2011, citado por Ribeiro, 2013) descubre diferentes rutas vinculadas con la inclusión digital y su concepto. La primera se relaciona con el "acceso", la segunda con "alfabetización digital" y la tercera con la apropiación de las tecnologías. 
Es decir, la inclusión digital comprende una extensión de variables necesarias para su definición, y cada una es un eje fundamental para que se pueda incluir a las personas en el mundo tecnológico.

Chacón, Ordoñez y Anichiarico (2017) plantean: "En este contexto emerge el concepto de inclusión digital, como una forma de inserción social imprescindible para el crecimiento de cualquier comunidad". (p.145). Por consiguiente, las personas de una comunidad o pueblo deben estar inmersas en temas y contenidos del área tecnológica, para que de esta manera sea una ventaja para el colectivo que habita la región.

En diferentes Estados a nivel internacional se tiene como política pública la inclusión digital, en Costa Rica se ha propuesto, aunque ha parecido quedar solo en papel. Chacón, Ordoñez y Anichiarico (2017) resaltan lo siguiente "se ha definido la inclusión digital como una política, o un conjunto de políticas que nacen del reconocimiento de la importancia de las TIC en la sociedad" (p. 145). Las tecnologías forman parte del mundo, así como la globalización, por lo que es un punto que se debe considerar como primordial para que las personas en las diferentes edades, específicamente con las personas de la tercera edad puedan tener su debido acceso.

Por su parte, mencionan Bisset, Grossi y Borsetti (2015): "En la Región de América Latina ... el acceso a las TIC's resulta ... insuficiente, si tenemos en cuenta que más del $50 \%$ la población la mayoría de los países aún no tiene acceso estas tecnologías" (p. 52). Considerando que las tecnologías son el medio principal que ha permitido la continuidad de la vida cotidiana de las personas hoy día, la desigualdad social en cuanto a la exclusión digital se traduce en un retroceso en el desarrollo. Esta condición impacta directamente el bienestar de la población, en este caso particular, de un sector de la población que se estima aumente significativamente en los próximos 9 años.

En el caso de Costa Rica, a pesar de los esfuerzos conjuntos de parte del Ministerio de Ciencia, Innovación, Tecnología y Telecomunicaciones y el Gobierno de Costa Rica (2015) aún no se consolida la inclusión digital como una política pública que vaya más allá de las palabras que soporta un documento. En este sentido, es necesario tomar acción, inyectar capital, generar proyectos en esta vía y contar con voluntad política para asegurar que todos los sectores de la sociedad tendrán acceso a dispositivos tecnológicos y contarán con los conocimientos que les permitirán utilizarlos significativamente en sus vidas. Según el II Informe de estado de la situación de la persona Adulta Mayor en Costa Rica (ESPAM), "se detectan casos por la incorrecta implementación o falta de recursos tecnológicos que han vulnerabilizado el acceso a otros derechos" (pp. 98-97). 
Las personas adultas mayores se están adentrando a una edad mucho más avanzada comparada con las personas que se encuentran en etapas anteriores, cuentan con características que las identifican como seres únicos e irremplazables. A su vez, adquieren variaciones en la parte física, psicológica e incluso social. La personas adultas mayores (en adelante AM), también conocidos como adultas tardías, según Mansilla (2000) son personas con aproximadamente 50 a 64 años, se ubican en el desarrollo biológico llamado climaterio. La mayoría de las personas dejan de trabajar y entran en la etapa de jubilación, por lo que en ese momento llegan a contar con tiempo disponible, así como con capacidad productiva existente.

Estas personas se encuentran en la etapa de envejecimiento, la cual es representada mediante diferentes aspectos que se proyectan en las personas que se aproximan a esta. De acuerdo con Papalia y Martorell (2017), la persona experimenta una serie de cambios durante esta etapa. En los físicos, la piel de la persona empieza a perder la elasticidad, palidecer y perder grasa, el cerebro los cambios pueden ser agudos, hacen poca diferencia en su funcionamiento, siempre y cuando las personas hayan tenido estilos de vida saludables. Algunas de las enfermedades que llegan a padecer son: cardiopatía, cáncer, apoplejía, diabetes, entre otras.

El área cognitiva es influenciada por la posición socioeconómica y el nivel educativo, asimismo la inteligencia psicométrica puede variar en cuanto a las condiciones que vivirán o han vivido las personas en la etapa adulta. Según Erikson, citado por Papalia y Martorell (2017), "el logro mayor de la adultez tardía es el sentido de integridad del yo, o integridad del sí mismo, un logro basado en la reflexión sobre la propia vida" (p. 529). A su vez, la misma autora menciona que las personas mayores necesitan evaluar y aceptar su vida para dar una apertura a la aceptación de la muerte.

\section{Referente contextual}

\section{Estrategia Nacional del para un Envejecimiento Saludable}

Por su parte, la Estrategia Nacional para un Envejecimiento Saludable se constituye como un esfuerzo de diversos ministerios públicos costarricenses, la Organización Panamericana de la Salud, la Organización Mundial de la Salud, así como la Universidad Nacional y la Universidad de Costa Rica, entre otras. Se trata de un documento en el cual se establecen las prioridades del Estado en relación con la población adulta mayor, en lo referente a garantizar sus derechos y la promoción de su bienestar. Persigue la mejora en la calidad de vida de esta población, así como el establecimiento de un modelo que promueva la salud y el envejecimiento saludable. 
En lo que se refiere al asunto que ocupa este artículo, dicha estrategia nacional plantea en varias ocasiones la necesidad de brindar acceso a la comunidad adulta mayor costarricense a tecnologías de asistencia, para incrementar sus capacidades y autonomía. Es así como se puede apreciar en el eje no 2 llamado "entornos saludables que favorezcan el envejecimiento", aquellos objetivos relacionados con el acceso a servicios tecnológicos para fomentar su autonomía, especialmente en lo que se refiere a los servicios públicos.

\section{El rol de las universidades públicas}

Las universidades públicas han desarrollado un rol elemental en cuanto al ejercicio de los derechos, por parte de la población adulta mayor. Así es como se puede destacar la labor del Programa de Atención Integral de la Persona Adulta Mayor (PAIPAM) de la Universidad Nacional (UNA). Este programa mantiene un enfoque inclusivo mediante el cual se imparten diversos cursos y proyectos de extensión que persiguen eliminar estereotipos negativos con respecto a la población adulta mayor, además se fomentan espacios de aprendizaje para el desarrollo integral de esta población.

Por su parte, la Universidad de Costa Rica (2017) realiza esfuerzos importantes por medio del Programa Institucional de la Persona Adulta y Adulta Mayor (PIAM). Se trata de un programa con más de 30 años de trayectoria, que ha llevado a cabo proyectos educativos, investigaciones, acción social y demás actividades para mejorar la calidad de vida de la población adulta mayor. Actualmente ofrece alrededor de 15 cursos orientados a esta población en lo referente a las tecnologías de la información y la comunicación, 11 cursos de idiomas, 18 cursos sobre movimiento humano y mucho más. Sus objetivos comprenden desde fomentar el diálogo intergeneracional, dotar de habilidades y competencias a la población adulta mayor, hasta la búsqueda de alianzas estratégicas para el mejoramiento de su calidad de vida.

También el Instituto Tecnológico de Costa Rica (TEC) posee el Proyecto Educativo para la Persona Adulta Mayor (PAMTEC). En este caso es ofrecido a personas de 55 años en adelante, y ofrece diferentes cursos que buscan que las personas de la tercera edad puedan disfrutar de un envejecimiento activo. Lo mismo sucede con la Universidad Nacional Estatal a Distancia (UNED) y la Universidad Técnica Nacional (UTN), instituciones de enseñanza superior que, a través de programas dirigidos a las personas adultas mayores, mejoran su calidad de vida y les proveen de capacidades para su diario vivir. 


\section{Metodología}

La investigación se desarrolla desde el paradigma naturalista cualitativo, al respecto conviene decir que tiene una gran influencia con el tema de recursos tecnológicos para las personas adultas mayores Desarrolla un análisis descriptivo desde diversos estudios. Para efecto de la presente investigación se va a trabajar desde el enfoque cualitativo.

Según Hernández, Fernández, y Baptista (2014) en este enfoque las personas investigadoras van a estar inmersas en el ambiente en el que se desenvuelve la población de interés. El estudio se llevará a cabo con diversas investigaciones relacionadas con las tecnológicas y las personas de la tercera edad. Los datos recolectados no serán basados en números, porcentajes o cifras, sino que el objetivo es explicar y describir el contexto en el que se desenvuelven estas personas con base en sus experiencias en cuanto a uso de las herramientas, mediante los datos arrojados en sus investigaciones científicas.

Mediante un diseño de investigación descriptiva (Hernández y Mendoza, 2018) se dan a conocer las características más elementales del fenómeno de estudio en el contexto actual. Además, se delimita el problema y su afectación en la población adulta mayor. De esta manera, mediante una investigación documental, se incluye información sobre los diversos elementos que componen el objeto de investigación y sus variables.

Para la producción de este artículo, se realizaron búsquedas de información en las bases de datos Ebsco Host de la Universidad Nacional y en la Revista Anales de Gerontología perteneciente a la Universidad de Costa Rica, así como búsquedas externas en repositorios de información de acceso libre tales como Kimuk, Kerwa, La Referencia, Latindex, Scielo, Redalyc, Dialnet, entre otras. Los datos estadísticos fueron obtenidos de fuentes primarias y secundarias.

Otros datos, de especial importancia, fueron tomados directamente del Ministerio de Salud de Costa Rica y cada uno de los sitios oficiales de los programas sobre envejecimiento que ejecutan las universidades públicas costarricenses. Una vez fue posible recolectar la información más atinente a la temática de esta investigación, se pudo analizar y así entender la dinámica actual. En total se analizaron 9 documentos de rigor científico. 


\section{Análisis e interpretación de datos}

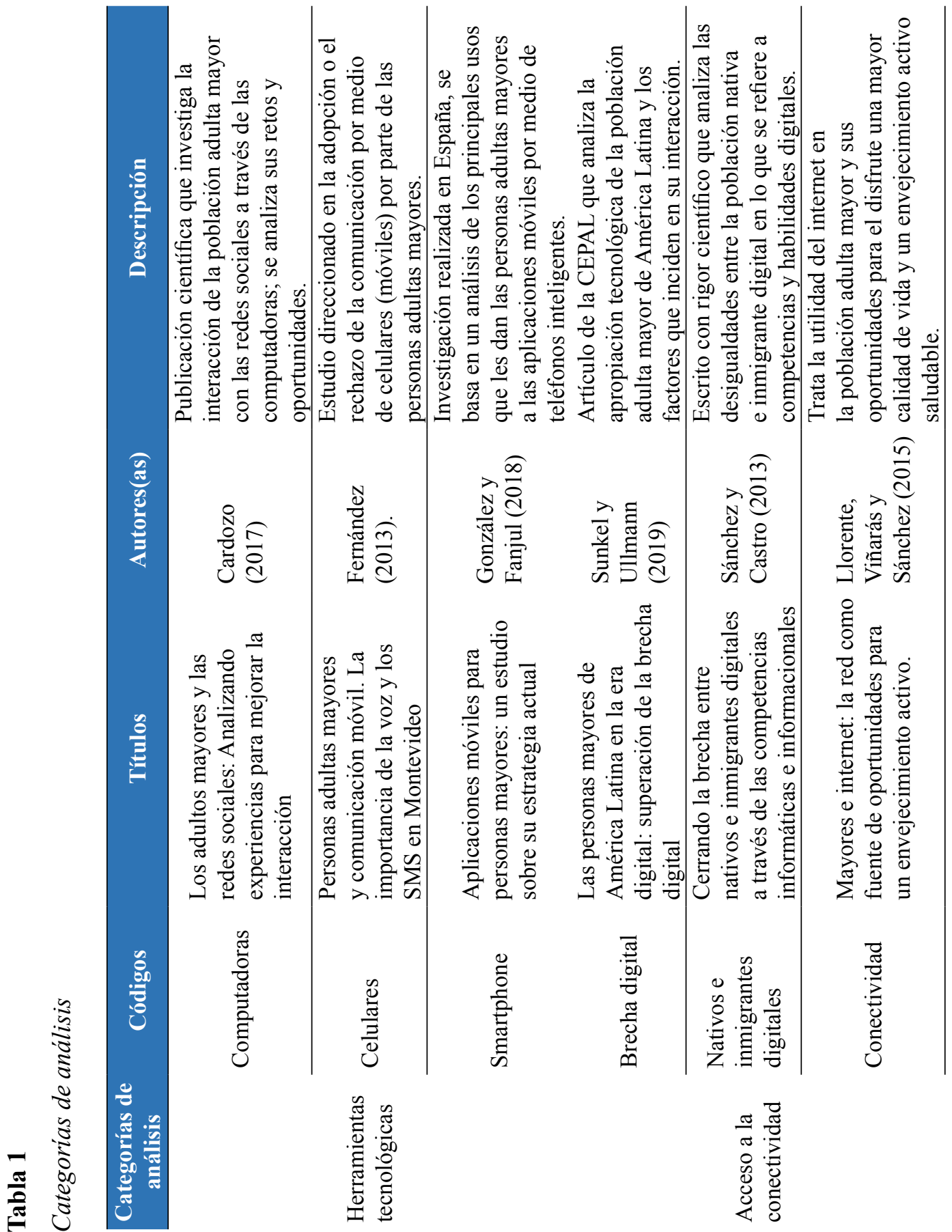




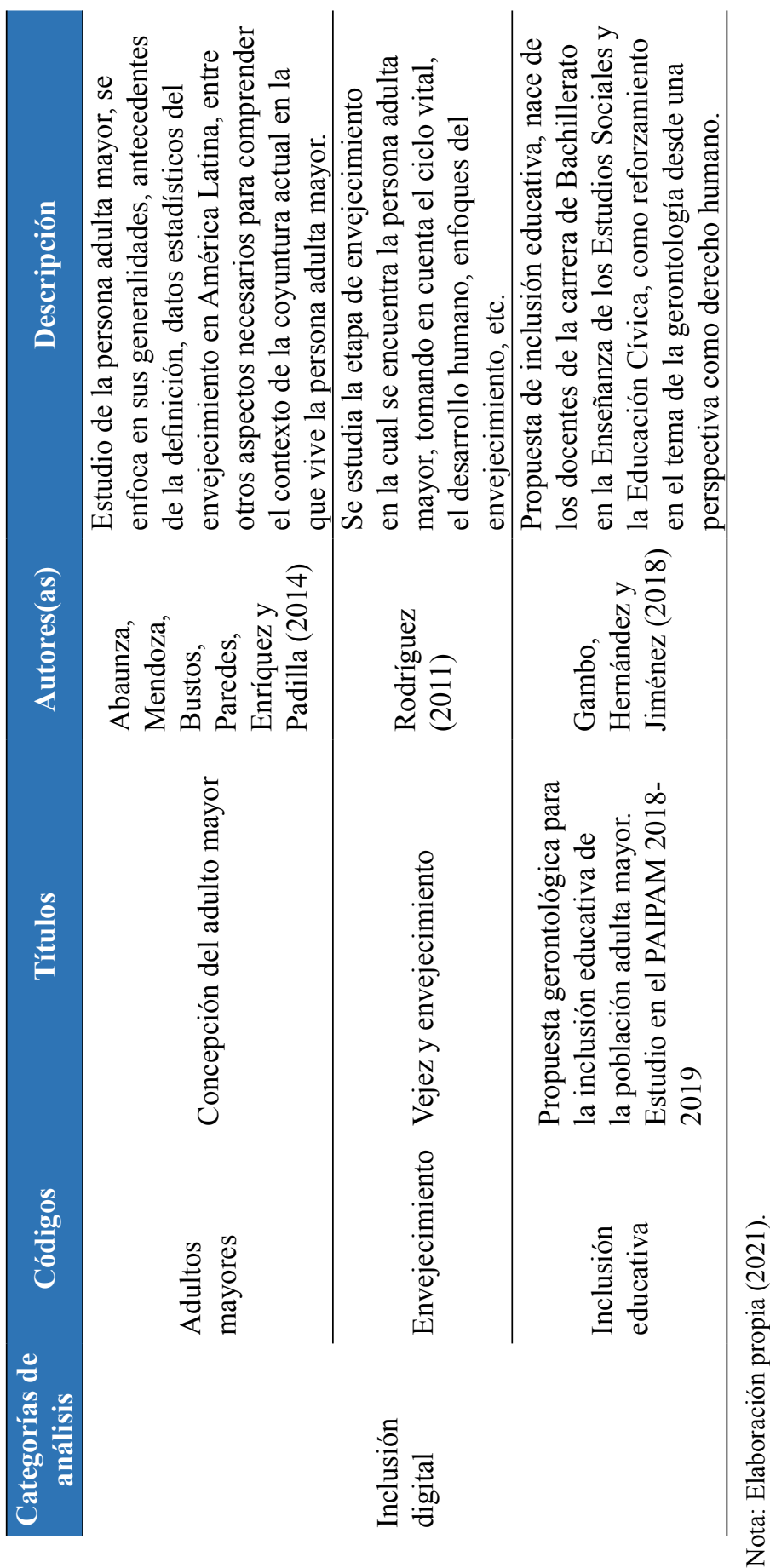

224 Revista Latinoamericana de Derechos Humanos 
Actualmente, los recursos tecnológicos se constituyen como los dispositivos preferidos para el acceso a la internet y, por tanto, a la información. Así lo entiende Cardozo (2017) en su obra titulada "Los adultos mayores y las redes sociales: Analizando experiencias para mejorar la interacción”. En una era digital, dominada por las TIC y en medio de una pandemia, el acceso a estos dispositivos toma un rol muy importante en cuanto a la calidad de vida de la población adulta mayor. Debido a lo anterior, el acceso a una computadora o teléfono inteligente significa la inclusión o exclusión de una sociedad que, desde hace tiempo, evolucionó mediante el uso de la tecnología.

Históricamente, las personas adultas mayores han sido consideradas como grupos vulnerables, es decir, que vivencian situaciones desventajosas debido a diversidad de condiciones y factores que los afectan con respecto a otros grupos sociales. En este sentido, el acceso a internet junto a la interacción del conglomerado adulto mayor, conforman un canal para generar bienestar en esta población. Hoy día, los recursos tecnológicos son la puerta al acceso a servicios y productos de una forma ágil y rápida.

Las redes sociales fungen como medios de comunicación que permiten a esta población mantenerse actualizada, disfrutar de su tiempo de ocio, ejecutar operaciones mercantiles, aprender y mantener el contacto con amigos y familiares. Uno de los grandes desafios se relaciona con el tema de la accesibilidad web, puesto que se hace necesario el desarrollo de aplicaciones y entornos digitales orientados a eliminar barreras de accesibilidad y que aseguren la integración de esta población.

Fernández (2013), en su estudio sobre "Personas adultas mayores y comunicación móvil. La importancia de la voz y los SMS en Montevideo" muestra un análisis sobre la repercusión que tienen los móviles en la persona adulta mayor. Es mediante la industria y las investigaciones académicas que este tema está siendo visible ante las diferentes ciencias y disciplinas científicas.

Los teléfonos inteligentes mayormente conocidos como (Smartphone) fueron investigados por González y Fanjul (2018) en su estudio "Aplicaciones móviles para personas mayores: Un estudio sobre su estrategia actual". Este es elaborado en España, señalado como país con un envejecimiento activo, donde se estima que para el 2050 el $32 \%$ de sus habitantes serán personas adultas mayores. Con el pasar del tiempo, la actualización e innovación de las tecnologías aumenta. En un tiempo determinado se llegó a tener teléfonos con difusión de mensajes y llamadas de texto, lo que aumentó la eficiencia de la comunicación. No obstante, los teléfonos de la actualidad contienen aplicaciones móviles que facilitan la vida cotidiana del ser humano. 
Los datos arrojados muestran que las personas adultas mayores conocen la aplicación de WhatsApp. Mientras que, por otra parte, dentro de las aplicaciones relacionadas con la salud física de los españoles conocen el podómetro, e identifican qué es la aplicación de diabetes. Sin embargo, muchas de las aplicaciones que son dedicadas a la persona adulta mayor no son conocidas por la desinformación existente sobre el tema.

Por otra parte, señalan Sunkel y Ullmann (2019) en su artículo "Las personas mayores de América Latina en la era digital: Superación de la brecha digital" que, si bien es cierto, los países de América Latina presentan distintas intensidades en el proceso de envejecimiento poblacional, la brecha digital continúa avanzando. Para Sunkel y Ullmann (2019), esto implica "incrementos proporcionales en las demandas sociales, económicas, políticas y culturales" (p. 245). Lo cierto es que las TIC se instituyen como precursores de la innovación y el desarrollo, en este caso, como una herramienta para combatir las desigualdades sociales e impulsar el progreso.

La brecha digital tiene variedad de manifestaciones: desde la falta de dispositivos electrónicos en el hogar, pasando por la imposibilidad de compra de estos y el escaso acceso a la conectividad, hasta el desconocimiento de su usabilidad. Son solo algunos de los factores que interfieren entre la persona adulta mayor y los recursos tecnológicos. Es de esta manera como se manifiesta la desigualdad, se acrecienta la brecha digital y toma mayor fuerza la exclusión social.

Una dinámica similar sucede con el factor edad, aunado al grado de dominio de competencias digitales en la sociedad. De esa forma, es posible dividir a la sociedad según sus habilidades y destrezas en entornos digitales, dando paso a la clasificación descrita por Prensky en 2001: las personas nativas e inmigrantes digitales. Generalmente, para la niñez, jóvenes y para cierta población adulta que ha sido alfabetizada digitalmente, el uso de las TIC parece ser algo que se trae incorporado; su facilidad de interacción con la tecnología no pareciese considerarse un problema para su vida y su uso se convierte en un elemento cotidiano.

Caso contrario sucede con la población adulta mayor, que se ve forzada a trasladarse de un universo analógico hacia uno digital y cuyas interacciones son mayormente mermadas, con respecto a los sujetos nativos digitales. En ese sentido, plantean Sánchez y Castro (2013) que cerrar "la brecha entre nativos e inmigrantes digitales a través de las competencias informáticas e informacionales" que se ven afectados debido a las limitaciones para navegar, acceder a la información y relacionar el mundo físico con el virtual, son problemáticas frecuentes a las que se enfrenta la población adulta mayor. Así, la alfabetización digital toma un rol destacado en 
aras a debilitar la desigualdad social y combatir la brecha existente entre personas nativas e inmigrantes digitales.

Por su parte, la conectividad a internet ofrece grandes oportunidades para esta población. Destacan Llorente, Viñaras y Sánchez (2015) en su obra "Mayores e internet" las siguientes: la red como fuente de oportunidades para un envejecimiento activo son la autosuficiencia, la autonomía, mejoras en procesos cognitivos y aspectos sociales. Es importante destacar que, a través del tiempo, entidades internacionales como las Naciones Unidas han intentado resolver esta situación, buscando la integración de todos los grupos sociales y fomentando así la cohesión social y productividad de los sectores más rezagados en esta área.

La población adulta mayor son el eje principal de este estudio, debido a que la idea principal es determinada por esta población. Abaunza, Mendoza, Bustos, Paredes, Enríquez y Padilla (2014) en su investigación titulada como "Concepción del adulto mayor" enmarcan que estas personas no se caracterizan por su edad, sino que se debe considerar el contexto de forma más analítica, es decir, tomar en cuenta los estilos de vida, la condición socioeconómica, los hábitos de vida saludables, entre otros.

El envejecimiento, según el estudio de Rodríguez (2011), titulado como "Vejez y envejecimiento", hace referencia al ciclo de vida, donde se señala que es el desarrollo integral del ser humano, tomando en cuenta diferentes áreas como la social, psicológica, física y biológica. El ciclo de vida involucra las diferentes etapas por las que transcurre el ser humano, desde la infancia, adolescencia, vida adulta y la vejez. En los tiempos antiguos, Grecia y Roma consideraban la vejez como un aspecto negativo, debido a que la muerte joven era un detonante de virtud, mientras que, por otra parte, Platón y Cicerón definen la vejez como etapa de experiencias recabadas por el ciclo vital. Sin embargo, es necesario que exista una inclusión en la educación de las personas adultas mayores, para que de esta forma puedan ser más autónomas.

Por último, Gamboa, Hernández y Jiménez (2018) realizan una propuesta con el nombre de "Propuesta gerontológica para la inclusión educativa de la población adulta mayor. Estudio en el PAIPAM 2018-2019". Se relata la importancia de un proceso colaborativo e incluyente para la persona adulta mayor. Su objetivo fue dar una herramienta para el personal facilitador del Programa de Atención Integral de la Persona Adulta Mayor de la Universidad Nacional de Costa Rica. Por lo tanto, lo anterior refleja una preocupación por parte de las universidades de educación superior por el deber de incluir a las personas adultas mayores en los procesos de enseñanza y aprendizaje. 


\section{Resultados y discusión}

El presente artículo de investigación deja en claro que el acceso y el uso de los recursos tecnológicos son elementos determinantes para la inclusión digital del público adulto mayor. De poco sirve que las entidades, tanto nacionales como internacionales, se preocupen por esta temática, si no se van a crear políticas públicas que verdaderamente contribuyan a combatir esta situación.

Con el paso de los años, a nivel mundial, se espera que la cantidad de personas adultas mayores crezca rápidamente, lo que trae consigo mayores demandas para los Estados. En lo que respecta a la conectividad a internet, lo ideal sería que se reconozca como derecho humano, de manera que sea un bien asequible para todas y todos por igual. Esta condición beneficiaría directamente a las personas adultas mayores en mayor desventaja social, dotándolas de una herramienta que, a la larga, contribuiría con un envejecimiento activo y saludable.

En general, cada una de las investigaciones consultadas mantuvo un eje focal: la importancia de incluir a la población adulta mayor en la sociedad para generar bienestar. Es importante mencionar que los medios sociales son un excelente punto de partida con el cual alfabetizar a este segmento poblacional, puesto que ofrecen facilidades prácticas en su vida cotidiana.

Se debe recalcar que, conforme pase el tiempo, la brecha digital en la comunidad adulta mayor se reducirá debido a que serán las nuevas generaciones quienes envejecerán a tal punto que, en un futuro, serán los grupos nativos digitales de las generaciones actuales las personas adultas mayores del mañana. Lo que se mantendrá en constante cambio son las necesidades, demandas e intereses de la población, por lo que se recomiendan programas de alfabetización digital orientados a satisfacerlas periódicamente.

Por otra parte, actualmente no se involucran a todas las entidades que tienen los recursos de infraestructura, de equipo y de recursos humanos para combatir la brecha digital, como lo son las bibliotecas públicas de cada comunidad. Dichas entidades tienen el potencial para fungir como centros de alfabetización digital de los pueblos aledaños a los que atienden. Una política pública en este sentido, que incluyera a estas entidades, se constituirá como un gran apoyo a los problemas descritos en este artículo; además por la naturaleza de las bibliotecas públicas, que siempre han servido a la sociedad.

Las tecnologías aún siguen en constante actualización, aspecto importante para la totalidad de integrantes y ciudadanía de una región. En la época actual, los recursos 
tecnológicos han tenido una fuerte incidencia en las actividades que realiza el ser humano, desde las domésticas, laborales e incluso de ocio. A su vez, han permitido aumentar la eficiencia en la comunicación entre las personas, sin importar el área geográfica. Dentro de los beneficios que enriquecen a las poblaciones están: medios de interacción social, facilidades ante la gestión de las diferentes empresas del mercado, facilidades para acceder a servicios online que brindan las instituciones públicas y privadas.

Sin embargo, es necesario que la accesibilidad sea para todas las personas por igual, incluyendo a las personas de la tercera edad. En las escuelas y los colegios, a nivel nacional e internacional, van implementando temas relacionados con los recursos tecnológicos, lo cual es una ventaja para las personas que se encuentran en la etapa de la infancia, niñez, adolescencia y adultez. Las intuiciones públicas y privadas se mantienen en una constante actualización durante todos los años para aprender sobre las diferentes herramientas novedosas que se lanzan en un mundo tan globalizado. Por lo tanto, se ha evidenciado en los diferentes documentos analizados en este estudio que el tema sobre el uso de los recursos tecnológicos está siendo estudiado e investigado.

Como se ha mencionado antes, la mayoría de los trámites en diferentes sectores institucionales son en línea, es decir, se requiere de acceso al internet y de un servidor para poder ser completado con éxito, ello representa una dificultad y vulnerabilidad para las personas de la tercera edad que no las saben utilizar, por lo que la inclusión educativa debe capacitar a estas personas para que se puedan dotar de conocimientos y aprendizajes que les pueda facilitar una adaptación ante la sociedad.

Los celulares son necesarios para una comunicación efectiva entre los seres humanos, a pesar de que no todas las personas pueden obtenerlos, las empresas especializadas en productos y servicios relacionados con estas herramientas intentan crear versiones que sean accesibles para todas las personas. Hace 20 años, aproximadamente, los primeros celulares tenían una singularidad, solo se utilizaban para mandar mensajes de texto y llamadas, lo que permitía que las personas que tenían familiares en el exterior se pudieran comunicar sin ningún problema.

En la actualidad, los celulares inteligentes son más eficientes y cuentan con sistemas que les permiten poder instalar diferentes aplicaciones que facilitan las tareas del diario vivir de las personas. La población adulta mayor, al igual que el resto de los miembros de la región, debe ser incluida en la era tecnológica para que logre obtener una mayor autonomía y comunicación con sus seres queridos. Por ejemplo, la Caja Costarricense de Seguro Social ahora cuenta con un expediente en línea, 
el cual es nombrado "EDUS", es mediante esta aplicación que la personas puede solicitar una cita, verificar los medicamentos, así como ver los registros médicos que se hacen a su nombre.

Aunado a lo anterior, si las personas adultas mayores fueran capacitadas en el uso de las herramientas tecnológicas, así como en el uso de las aplicaciones que son creadas para que todos los seres humanos a nivel integral, sin exclusiones, puedan utilizar y acceder a ellas, su vida sería mayormente sencilla.

La vejez no es una limitación para aprender, dependiendo del contexto en el que viven las personas adultas mayores, pueden ser formadas de manera eficiente con los recursos tecnológicos. Como se argumenta en los cambios físicos, psicosociales, psicológicos, entre otras, estas personas pueden tener un aprendizaje significativo y permanente que les permita poder crecer como seres humanos independientes e incluidos en una sociedad tan cambiante.

\section{Conclusiones}

Diferentes estudios han mostrado que es importante incluir a las personas adultas mayores en el nuevo mundo digital. Los recursos tecnológicos forman parte de la vida personal-social del ser humano, por lo que es indispensable que todas las personas a nivel integral puedan tener acceso a estas, así como brindar capacitaciones que sean fomentadas desde el Estado y las diferentes instituciones públicas y privadas, de modo que estas personas pueden tener una vida mucho más independiente, con una comunicación más eficaz e interactiva.

A su vez, es importante que no se denigre a la persona adulta mayor por su edad o deterioro físico y cognitivo, sino que se puedan atender como seres humanos de manera integral. Como lo demuestran diferentes estudios, las personas adultas mayores, con estilos de vida saludables y con buenos hábitos, tienen las capacidades de aprendizajes suficientes para que se les capacite. No obstante, si existiese alguna dificultad de discapacidad, también deben ser integradas e incluidas al igual que con los demás individuos, porque son seres humanos que cuentan con los mismos derechos que toda la ciudadanos y habitantes de un país.

La inclusión digital concebida como política pública en Costa Rica fue un tema que se trató en el Plan Nacional de Desarrollo de las Telecomunicaciones 2015-2021, sin embargo, parece quedar únicamente en palabras, puesto que las acciones orientadas a remediar la situación solo quedaron dentro del documento. Como respaldo de lo anteriormente dicho, el acto más reciente de la Asamblea, al no haber aprobado el Programa Nacional de Alfabetización Digital, lo que solo deja en evidencia que 
los intereses políticos particulares privan por sobre todas las demás necesidades de la sociedad. También, esta investigación documental sirve para dilucidar que la población adulta mayor requiere de mayores oportunidades para conseguir incluirse satisfactoriamente en la sociedad actual, dominada por las tecnologías.

Tanto los antecedentes como el desarrollo del artículo exponen la realidad social que vive la población adulta mayor en materia de inclusión digital; expone, así, la importancia de cubrir las necesidades tecnológicas de este grupo social relacionadas a su acceso y uso para beneficiarlo con un envejecimiento saludable, inclusivo y activo. En este sentido, los grupos profesionales de las distintas áreas del conocimiento que se vean inmersas en este tema, estan llamados a responder a las demandas propias de la cuarta revolución industrial con la intención de evitar dejar a alguno de los diversos grupos sociales en la exclusión. Finalmente, resulta oportuno mencionar que, si el Estado no es consciente de los beneficios de contar con altos índices de alfabetización digital en la población, se carece de voluntad política, así como de recursos financieros para dotar de acceso y uso a los recursos tecnológicos a la sociedad, lo ideal sería que al menos se sienten las bases para proyectos futuros en esta vía.

\section{Referencias}

Abaunza, C., Mendoza, M., Bustos, P., Paredes, G., Enríquez, K. y Padilla, A. (2014). Concepción del adulto mayor: Adultos mayores privados de la libertad en Colombia. DOI: https://doi.org/10.7476/9789587385328.0007

Arias, M., Sandia, B., Mora, E. (2012). La didáctica y las herramientas tecnológicas web en la educación interactiva a distancia. Educere, 16(53), 21-36. https://n9.cl/xaqp

Banco Mundial. (2019). Esperanza de vida al nacer, total (años) - Costa Rica. https:// $\mathrm{n} 9 . \mathrm{cl} / \mathrm{sh} 7 \mathrm{r}$

Benavides, A. y Chipana, Y. (2021). Competencias digitales en adultos mayores y acceso a la justicia: Una revisión sistemática. Revista de Derecho, 6(1), 182-194. https://doi. org/10.47712/rd.2021.v6i1.121

Bisset, E., Grossi, A. y Borsetti, S. (2015). Políticas públicas de inclusión digital: El caso de América Latina y Cuba. Biblios: Journal of Librarianship and Information Science, (58), 42-53. https://doi.org/10.5195/biblios.2015.203

Cardozo, C. (2017). Los adultos mayores y las redes sociales: Analizando experiencias para mejorar la interacción. Informes Científicos Técnicos de la Universidad Nacional de la Patagonia Austral, 9(2), 1-29. https://doi.org/10.22305/ict-unpa.v9i2.244 
Centro de Estudios Generales. (2015). Programa de Atención de la Persona Adulta Mayor (PAIPAM). https://n9.cl/mlkh9

Chacón, Á., Ordóñez, J., Anichiarico, A. (2017). Hacia el reconocimiento de la inclusión digital como un derecho fundamental en Colombia. Vniversitas, (134), pp. 139-168. https://doi.org/10.11144/Javeriana.vj134.hrid

Consejo Nacional de la Persona Adulta Mayor. (2013). Política Nacional de Envejecimiento y Vejez 2011 - 2021. CONAPAM. https://n9.cl/bnhjd

Corrales, I. y Sánchez, Y. (2018). El aprendizaje cognoemocional en un grupo de personas mayores. Anuales en Gerontología, (10), 159-169. https://n9.cl/4zq9n

Estado Actual de la Persona Adulta Mayor. (2020). II Informe estado de situación de la persona adulta mayor en Costa Rica. Universidad de Costa Rica. https://n9.cl/3klb

Fernández, M. (2013). Personas adultas mayores y comunicación móvil. La importancia de la voz y los SMS en Montevideo. Revista de Ciencias Sociales, 26(33), 97-120. https://n9.cl/2fmpw

Flores, L. (2020). La alfabetización digital en el público adulto mayor: Un acercamiento desde la comunicación de las relaciones públicas en Perú. Revista ConHumanitas, 11(2), 65-80. https://doi.org/10.31207/rch.v11i2.239

Gamboa, D., Hernández, C. y Jiménez, H. (2018). Propuesta gerontológica para la inclusión educativa de la población adulta mayor. Estudio en el PAIPAM 2018-2019 [Tesis de Licenciatura]. Universidad Nacional. https://n9.cl/k9ad4

Gonzales, C. y Fanjul, C. (2018). Aplicaciones móviles para personas mayores: Un estudio sobre su estrategia actual. Aula Abierta, 47(1), 107-112. https://doi.org/10.17811/ rifie.47.1.2018.107-112

Hernández, R., Fernández, C. y Baptista, P. (2014). Metodología de la investigación (6 ${ }^{\mathrm{a}}$ ed.). McGraw Hill Education.

Hernández, R. y Mendoza, C. (2018). Metodología de la investigación: Las rutas cuantitativa, cualitativa y mixta. McGraw-Hill Interamericana Editores.

Huenchuan, S. (2019). Envejecimiento, personas mayores y Agenda 2030 para el Desarrollo Sostenible: Perspectiva regional y de derechos humanos. Comisión Económica para América Latina y el Caribe. https://doi.org/10.18356/19532890-es

Instituto Nacional de Estadística y Censos. (2011). Estadísticas demográficas. 2011 - 2025. Proyecciones nacionales. Población total proyectada al 30 de junio por grupos de edades, según provincia y sexo [Hojas de Excel]. https://n9.cl/1ckkn 
Llorente, C., Viñaras, M. y Sánchez, M. (2015). Mayores e internet: La red como fuente de oportunidades para un envejecimiento activo. Revista Cientifica de Educomunicación, 23(45), 29-36. https://n9.cl/5behh

Mansilla, M. (2000). Etapas del desarrollo humano. Revista de investigación en Psicología, 3(2), 105-116. DOI: https://doi.org/10.15381/rinvp.v3i2.4999

McLuhan, M. (2013). Componentes básicos de una computadora. https://n9.cl/jexg

Ministerio de Ciencia, Innovación, Tecnología y Telecomunicaciones y Gobierno de Costa Rica. (2015). Plan nacional de desarrollo de las telecomunicaciones 2015 - 2021. https://n9.cl/77sn4

Ministerio de Salud de Costa Rica. (2018). Estrategia nacional para un envejecimiento saludable basado en el curso de vida 2018 - 2020. https://n9.cl/9e50

Organización para la Cooperación y el Desarrollo Económicos. (2020). Aprovechar al máximo la tecnología para el aprendizaje y la formación en América Latina. OCDE. https://doi.org/10.1787/ce2b1a62-en

Papalia, D. y Matorell, G. (2017). Desarrollo humano. (12. ed.). Editorial McGraw-Hill/ Interamericana.

Prensky, M. (2001). Digital natives, digital immigrants. On The Horizon, 9(5), 1-6. https:// doi.org/10.1108/10748120110424816

Ramírez, L. y Sepúlveda, J. (2018). Brecha digital e inclusión digital: Fenómenos socio - tecnológicos. Revista EIA, 15(30), 89-97. https://doi.org/10.24050/reia.v15i30.1152

Ribeiro, F. (2013). Inclusión digital como política pública: Disputas en el campo de los derechos humanos. Revista Internacional de Derechos Humanos, 10(18), 33-55. https:// n9.cl/61r $2 \mathrm{p}$

Rivera, R y Urbina, B. (2019). Proceso de alfabetización tecnológica en los adultos y adultos mayores participantes del proyecto de extensión manejo básico de oficinas de la carrera educación comercial, universidad nacional, periodo 2012-2016 [Tesis de Licenciatura]. Universidad Nacional de Costa Rica. Repositorio Institucional. https:// n9.cl/wvv1o

Rodríguez, K. (2011). Vejez y envejecimiento. Doc. Inv. Esc. Med. Cs. Salud, (12), 1-43. https://n9.cl/tlrg5

Rodríguez, O., Hernández, R., Torno, L., García, L., Rodríguez Romero, Roland. (2005). Telefonía móvil celular: Origen, evolución, perspectivas. Ciencias Holguín, 11(1), 1-8. https://n9.cl/t3pv 
Ruiz, M. (2015). Herramientas tecnológicas como instrumentos para la gestión del conocimiento en las organizaciones cooperativas. Estrategias, 12(22), 6-15. https://doi. org/10.16925/es.v12i22.960

Saavedra, C. (2014). Una mirada a los estilos de aprendizaje de los estudiantes de la UPTC desde la noción del nativo digital. Revista Academia y Virtualidad, 7(2), 41-52. https:// doi.org/10.18359/ravi.317

Sánchez, A. y Castro, D. (2013). Cerrando la brecha entre nativos e inmigrantes digitales a través de las competencias informáticas e informacionales. Apertura, 5(2), 1. https:// n9.cl/6r0sv

Sistema Nacional de Vigilancia Epidemiológica Fitosanitaria de México. (SINAVEF). (2011). Smartphone. https://n9.cl/f48yd

Sunkel, G. y Ullmann, H. (2019). Las personas mayores de América Latina: Superación de la brecha digital. Revista de la CEPAL, (127), 243-268. https://doi.org/10.18356/ db143bd3-es

Universidad de Costa Rica. (2017). Programa Institucional para la Persona Adulta y Adulta Mayor. https://n9.cl/zzv7 